\title{
Laryngeal Injury
}

National Cancer Institute

\section{Source}

National Cancer Institute. Laryngeal Injury. NCI Thesaurus. Code C35395.

Trauma to the larynx. 